\title{
dc breakdown conditioning and breakdown rate of metals and metallic alloys under ultrahigh vacuum
}

\author{
A. Descoeudres, ${ }^{*}$ T. Ramsvik, S. Calatroni, M. Taborelli, and W. Wuensch \\ European Organization for Nuclear Research, CERN, 1211 Geneva 23, Switzerland
}

(Received 8 January 2009; published 24 March 2009)

\begin{abstract}
The rf accelerating structures of the Compact Linear Collider (CLIC) require a material capable of sustaining high electric field with a low breakdown rate and low induced damage. Because of the similarity of many aspects of dc and rf breakdown, a dc breakdown study is underway at CERN in order to test candidate materials and surface preparations, and have a better understanding of the breakdown mechanism under ultrahigh vacuum in a simple setup. Conditioning speeds and breakdown fields of several metals and alloys have been measured. The average breakdown field after conditioning ranges from $100 \mathrm{MV} / \mathrm{m}$ for $\mathrm{Al}$ to $850 \mathrm{MV} / \mathrm{m}$ for stainless steel, and is around $170 \mathrm{MV} / \mathrm{m}$ for $\mathrm{Cu}$ which is the present base-line material for CLIC structures. The results indicate clearly that the breakdown field is limited by the cathode. The presence of a thin cuprous oxide film at the surface of copper electrodes significantly increases the breakdown field. On the other hand, the conditioning speed of Mo is improved by removing oxides at the surface with a vacuum heat treatment, typically at $875^{\circ} \mathrm{C}$ for 2 hours. Surface finishing treatments of $\mathrm{Cu}$ samples only affect the very first breakdowns. More generally, surface treatments have an effect on the conditioning process itself, but not on the average breakdown field reached after the conditioning phase. In analogy to rf, the breakdown probability has been measured in dc with $\mathrm{Cu}$ and Mo electrodes. The dc data show similar behavior as $\mathrm{rf}$ as a function of the applied electric field.
\end{abstract}

DOI: 10.1103/PhysRevSTAB.12.032001

PACS numbers: 52.80.Vp, 52.80.Pi, 29.20.Ej

\section{INTRODUCTION}

The feasibility of the future $12 \mathrm{GHz}$ multi-TeV $e^{+} e^{-}$ Compact Linear Collider (CLIC) is under investigation at CERN [1-4]. In order to limit this linear collider to an acceptable length, extremely high accelerating gradients of the order of $100 \mathrm{MV} / \mathrm{m}$ are required. With such fields, rf breakdowns are likely to occur and produce damage on the accelerating cavities. Therefore, a material capable of sustaining high electric fields with low damage after breakdowns is needed. Furthermore, during operation, breakdowns can also lead to the loss of the accelerated beam due to random kicks. Thus, each structure must have a breakdown probability (or breakdown rate) as low as possible, typically in the order of $10^{-7}$ breakdowns per rf pulse.

In this context, a dc breakdown study is underway at CERN in order to test candidate materials and surface preparations, and also to have a better comprehension of the breakdown mechanism under ultrahigh vacuum [5-8]. The dc tests are fast, more flexible, and are more easily instrumented than high power rf tests, and can be performed with a much simpler setup. The results obtained with this experiment, run in parallel to rf structure tests, are therefore useful to get information about the physical quantities governing breakdown and electrode damage, and to have additional inputs for the design and the choice

*antoine.descoeudres@cern.ch of materials for future high gradient accelerating structures.

Vacuum breakdowns have been studied for many years in other areas, such as vacuum circuit breakers $[9,10]$ and more recently in dc guns for $\mathrm{x}$-ray free electron lasers (XFEL), for example [11]. Theories of vacuum breakdown exist [12-16], but a complete understanding of this phenomenon is still lacking. Also many issues such as breakdown rate are specific to linear colliders and have thus not been measured.

Measurements of conditioning speeds and breakdown fields of several metals and metallic alloys are presented in this paper. Copper and molybdenum have been more specifically investigated, since $\mathrm{rf}$ tests are presently focused on structures built of these metals. Copper is obviously interesting for rf applications due to its high electrical conductivity and the possibility to machine it easily and precisely, whereas molybdenum has a high melting point with a relatively good electrical conductivity at the same time. The effects of vacuum heat treatments on breakdown fields of these two metals have been investigated, as well as different surface finishing treatments on copper. The dc breakdown rate measurements have also been performed on $\mathrm{Cu}$ and $\mathrm{Mo}$, and the results are compared to the rf data.

\section{EXPERIMENTAL SETUP}

Figure 1 shows a schematic view of the dc spark setup. Both electrodes are made of the same material unless 


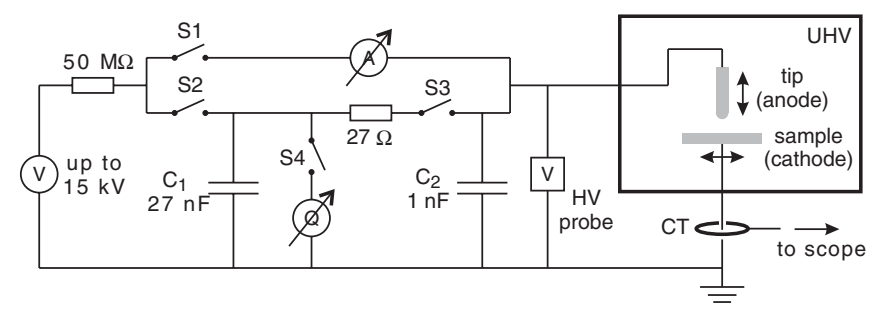

FIG. 1. Schematic drawing of the experimental setup.

explicitly specified, in a point-to-plane configuration. They are located in an ultrahigh vacuum (UHV) chamber at a typical pressure of $5 \times 10^{-10}$ mbar. When bakeouts are performed to reach UHV, the vacuum chamber is heated to typically $150^{\circ} \mathrm{C}$ for 24 hours. The anode is a hemispherical rounded tip, $2 \mathrm{~mm}$ in diameter, and the cathode (sample) is a grounded $10 \mathrm{~mm} \times 50 \mathrm{~mm}$ rectangular plane surface, $2 \mathrm{~mm}$ in thickness. All samples are directly cut from polycrystalline cold rolled sheets and tips are obtained by turning the end of cylindrical rods. Unless explicitly specified, no prior heat treatments are applied to the electrodes. The sample can be moved laterally inside the chamber in order to test several spots at its surface. The vertical position of the tip, and therefore the gap distance, is controlled with a micropositioning device combined with differential levers. The positioning accuracy of this system is around $1 \mu \mathrm{m}$, and the gap distance is set typically to $20 \mu \mathrm{m}$. Such small gaps are necessary to reach fields of several hundreds of $\mathrm{MV} / \mathrm{m}$ with the available $15 \mathrm{kV}$ power supply. The zero distance is found by bringing the electrodes into contact and measuring a short circuit. In the present geometrical configuration and with a $20 \mu \mathrm{m}$ gap distance, the field profile on the cathode surface is slightly decreasing with the distance from the symmetry axis: the field reaches $90 \%$ of its peak value at $70 \mu \mathrm{m}$ from the axis, and $80 \%$ at $100 \mu \mathrm{m}$, for example.

Field emission (FE) measurements between the electrodes can be performed by closing the $S 1$ relay and by applying high voltage to the anode directly from the power supply. The FE current is read with a multimeter. From these current-voltage characteristics and the assumption that they follow a Fowler-Nordheim behavior, the field enhancement factor $\beta$ can be calculated [5]. For the measurement of the breakdown field $E_{b}$, the $27 \mathrm{nF}$ capacitor $C_{1}$ is charged with the power supply first to a low value via the relay $S 2$, and then connected to the anode via the high current relay $S 3$ for typically two seconds. If no breakdown occurs, the voltage is increased and the cycle is repeated until the breakdown field is reached. Sparks are repetitively produced in this way in order to condition the tested spots on the electrode surfaces. The accuracy of the gap distance is checked before and several times during a conditioning experiment by reestablishing contact between the two electrodes. Breakdowns are detected either by measuring the remaining charge on the capacitor after the exposure time by closing the relay $S 4$, or simply with a $500 \mathrm{MHz}$ current transformer (CT) connected to a $1 \mathrm{GHz}$ scope. The $C_{2}$ capacitor is used to damp voltage overshoots when the $S 3$ switch is closed. Time delays between the voltage rise and a breakdown can be measured with a $75 \mathrm{MHz}$ high voltage probe, also connected to the scope. For breakdown rate measurements, the main capacitor $C_{1}$ is always charged at the same voltage and eventual breakdowns are detected after each attempt with the current transformer. In the present setup, the maximal energy available for the discharge is around $1 \mathrm{~J}$ and is chosen to be of the same order as in the rf experiments at $30 \mathrm{GHz}$ conducted at CERN. More details about the setup can be found in [5].

Several pure metals and metallic alloys have been tested: Al (Anticorodal 100-112), C (graphite 2020 PT), Cr (99.99\% purity), $\mathrm{Cu}$ (OFE, UNS C10100), Mo (99.95\% purity), $\mathrm{Nb}$ (99.9\% purity), Ta (99.9\% purity), Ti (99.6\% purity), V (99.8\% purity), W (99.95\% purity), Glidcop ${ }^{\circledR}$ (AL-15 C15715), copper-zirconium alloy (C15000), tungsten carbide (89\%WC-10\%Co, $0.8 \mu \mathrm{m}$ grain size), Ti$15 \% \mathrm{Mo}$ (R58150), and stainless steel (316LN). With the exception of carbon, all tested materials are cleaned according to the CERN standard procedure for UHV components [17] prior to installation in the UHV chamber. Molybdenum is in addition chemically etched to remove some oxides. The carbon (graphite) electrodes were cut by milling. The tip and sample were then cleaned by ultrasonic bath of ethanol, followed by $\mathrm{CO}_{2}$ dry ice blasting.

\section{RESULTS AND DISCUSSION}

\section{A. Conditioning and saturated breakdown fields}

Figure 2 shows the evolution of the breakdown field $E_{b}$ for some of the tested pure metals. A conditioning phase is generally observed, during which the breakdown field increases with the successive sparks until it reaches saturation. The saturated field $\bar{E}_{b}$ is calculated by taking the average of the breakdown fields after the conditioning phase, where saturation occurs. The number of sparks necessary to reach saturation depends on the material, but is typically between 20 and 100 . Copper behaves differently than the other tested materials, since saturation is reached immediately ("immediate conditioning"). The scattering in the values of $E_{b}$ in the saturation region is caused by continuous surface modifications due to sparks, leading either to improved or worsened resistance to breakdown. Therefore the saturated field, which is an averaged value, slightly underestimates the ultimate breakdown field of the material, which corresponds rather to the highest values reached in the saturation region. Stable operation below the ultimate breakdown field is addressed in Sec. III D.

Although titanium has the highest saturated field of all metals tested here, it shows nevertheless a strong erosion and material displacement after breakdowns, so that the gap spacing must be continuously readjusted. A decrease 
(a) $\mathrm{C}$

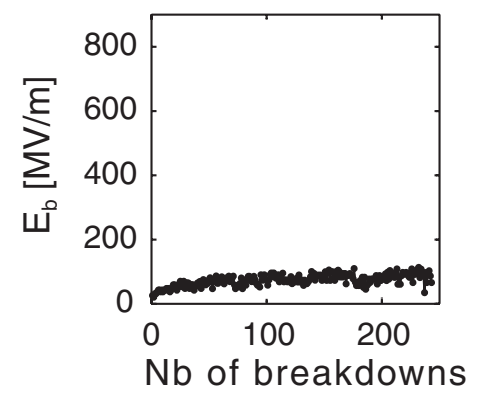

(e) Mo

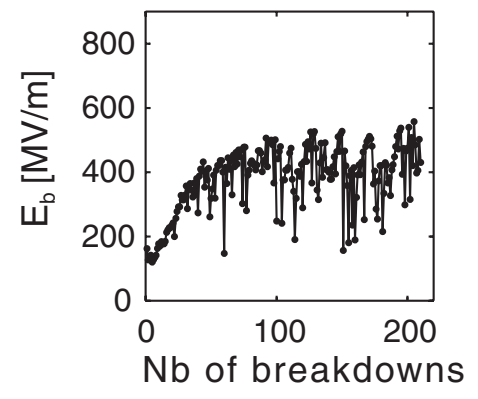

(b) $\mathrm{Cu}$

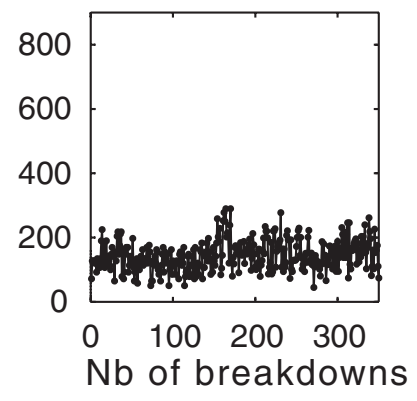

(f) $\mathrm{Cr}$

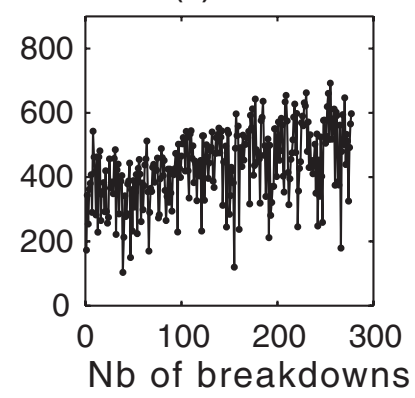

(c) W

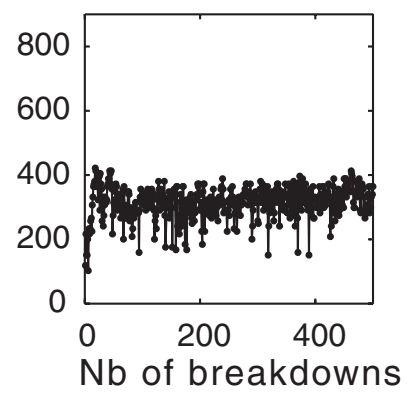

(g) $\mathrm{V}$

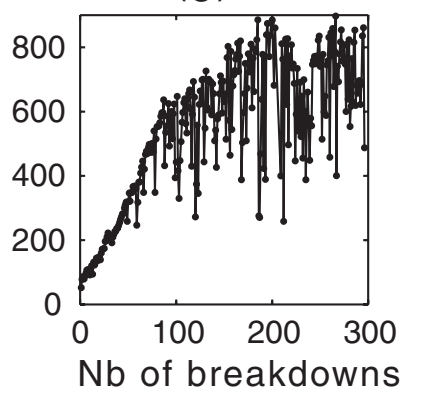

(d) $\mathrm{Nb}$

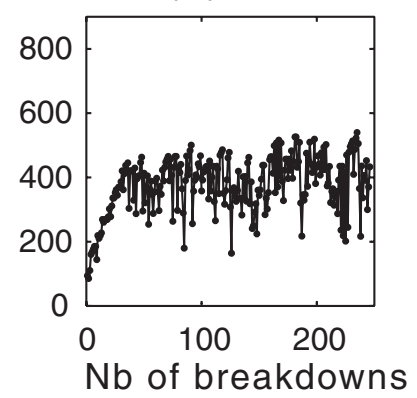

(h) $\mathrm{Ti}$

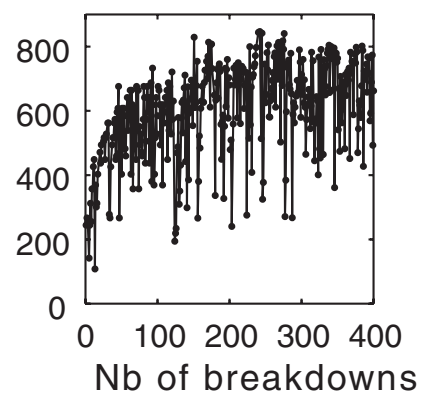

FIG. 2. Conditioning curves of pure metals. The metals are ranked according to their saturated breakdown field.

or an increase in the gap distance up to $\pm 50 \%$ of the original gap distance can be observed after a few tens of sparks. Gaps with $\mathrm{Cu}$ or Mo electrodes are more stable, for example: variations are typically smaller than $\pm 10 \%$ after 50 breakdowns. All values of $E_{b}$ presented in this paper are corrected taking into account these changes in the gap distance occurring during the experiments.

Since the mechanical properties of pure $\mathrm{Cu}$ can be improved by alloying, especially its tensile and fatigue strengths, copper-based alloys are of interest in the CLIC context [18]. As shown in Figs. 3(a) and 3(b), the two copper-based materials tested in this study, $\mathrm{CuZr}$ alloy and Glidcop $(\mathrm{Cu}$ matrix with submicroscopic alumina particles), perform slightly worse than pure $\mathrm{Cu}$ in terms of reached breakdown field. As for $\mathrm{Cu}$, they also show an immediate conditioning. The difference in $E_{b}$ is around (a) $\mathrm{CuZr}$

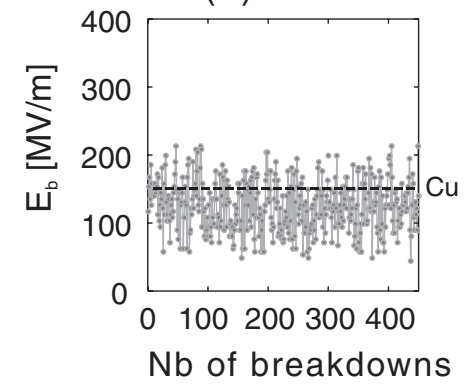

(b) Glidcop

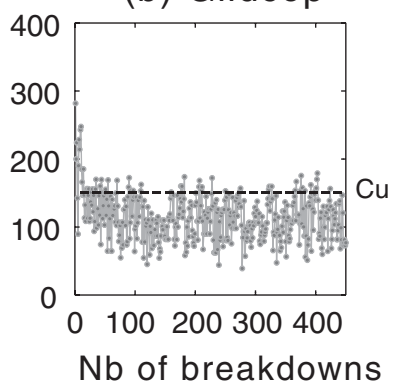

FIG. 3. Conditioning curves of copper-based materials.
$-30 \%$ compared to $\mathrm{Cu}$. Tensile and fatigue strengths are macroscopic properties of materials, therefore they have probably a small influence on the breakdown field measurements, which are done on a microscopic scale. Moreover, the macroscopic properties of these alloys could also be lost due to the local melting of the surface.

Figure 4 shows the conditioning curves of the other tested alloys. Although being much harder, tungsten carbide behaves similarly to pure tungsten [Fig. 4(a)]. As observed with copper-based materials, a higher macroscopic hardness does not necessarily imply a higher resistance to breakdown. The Ti-Mo alloy, which could potentially combine the high saturated field of $\mathrm{Ti}$ with the smaller gap variability of Mo, tends to be even more fragile than pure titanium. High fields are also reached [Fig. 4(b)], but the gap distance is extremely unstable. Gap variations up to $\pm 100 \%$ can be observed after a single spark. This leads also to the large spread of the $E_{b}$ values observed, and the calculation of an average breakdown field is irrelevant in these conditions. This fragility implies that severe damage is likely to occur in rf structures built of this material. On the contrary, stainless steel is an interesting candidate for accelerating structures. As shown in Fig. 4(c), this material has the largest saturated field of all materials tested so far $(850 \mathrm{MV} / \mathrm{m})$. The conditioning is fast (20 sparks) and the gap variability is similar to those of $\mathrm{Cu}$ and $\mathrm{Mo}$, for example. But since the electrical conductivity of stainless steel (SS) is rather low, bimetallic structures $\mathrm{Cu} / \mathrm{SS}$ are preferred to pure SS structures for practical rf applications. The first rf tests of such structures 
(a) W-C

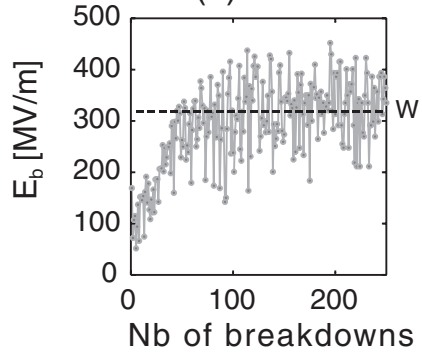

(b) Ti-Mo

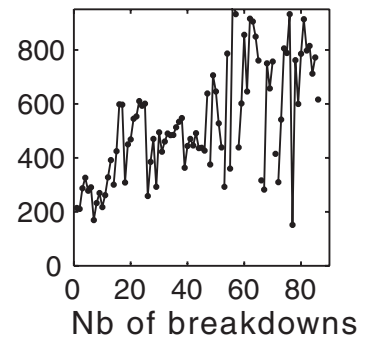

(c) SS

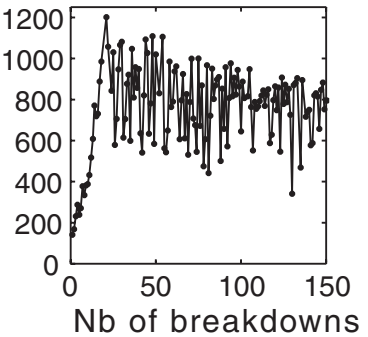

FIG. 4. Conditioning curves of other alloys: tungsten carbide, titanium-molybdenum, and stainless steel.

have recently been reported [19], as well as tests of $\mathrm{Cu}$ structures with clamped Mo and W irises [20,21].

In this context, electroplating is a possible technique to deposit a material with a higher breakdown resistance on a copper substrate. A copper sample with a $500 \mu \mathrm{m}$ thick chromium coating has been tested. Early tests with coatings in the $\mu \mathrm{m}$ range showed that such thin coatings are rapidly destroyed by a few breakdowns. After deposition, the surface has been ground to reduce the roughness, and cleaned following the standard procedure. The dc breakdown tests have been done with a chromium tip. As expected, the $E_{b}$ values measured are those typically obtained with pure $\mathrm{Cr}$ electrodes.

The saturated breakdown field of all tested materials is summarized in Fig. 5. It is clear from this ranking that many physical quantities are involved in the breakdown process. For example, refractory metals are not necessarily better than others. The ranking cannot be explained by only one dominant material property, but rather by a complex combination of several ones such as melting point, heat of fusion, thermal conductivity, electrical conductivity, vapor pressure, surface tension, and work function, for example. In $30 \mathrm{GHz}$ rf experiments, the ranking of copper, tungsten,

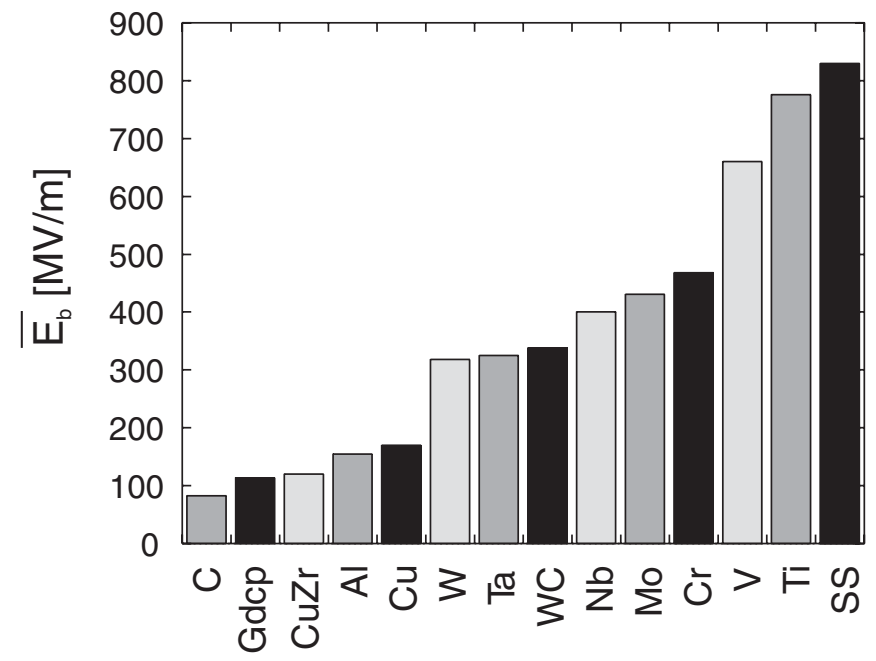

FIG. 5. Average breakdown field after conditioning of the main materials tested. and molybdenum is the same as in this de study, if one considers the high breakdown rate data for rf [22]. It should be noted that the values in Fig. 5 are valid for the geometry of the present experiment, with the specified separation between electrodes $(20 \mu \mathrm{m})$. Modifying the interelectrode distance can lead to different values [14,23]. Moreover, the rf breakdown field is known to depend also on the pulse length, and therefore the values of $E_{b}$ in dc and $\mathrm{rf}$ can be different.

In order to distinguish whether the breakdown field is limited by the anode or the cathode, experiments were performed with electrodes of different materials facing each other. Figure 6 shows that the breakdown field in the configuration with a tungsten cathode and titanium anode corresponds to the value found for a system with both electrodes of tungsten [Fig. 2(c)]. For the configuration having a titanium cathode and a tungsten anode, the measured breakdown field is that of titanium [Fig. 2(h)]. This proves that the resistance to breakdown is limited by the cathode in this case. This result is in good agreement with many UHV breakdown models, which emphasize the crucial role of field emission, originating from the cathode, in the initiation of the breakdown mechanism [12-16]. Indeed, in the present geometry with gaps in the 1020 micrometer range, the fact that $E_{b}$ is limited by the cathode can be explained by the low energy deposited in the anode. At equal electric field, a smaller gap needs a lower voltage, and field emitted electrons will deposit only a moderate amount of energy on the anode. The heating (a) Ti anode - W cathode

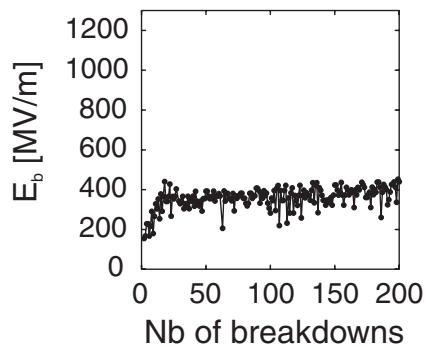

(b) W anode - Ti cathode

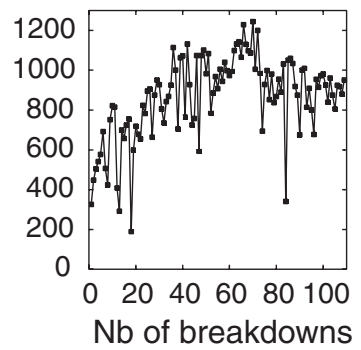

FIG. 6. Conditioning curves for: (a) Ti anode and W cathode; (b) W anode and Ti cathode. 
(a) untreated

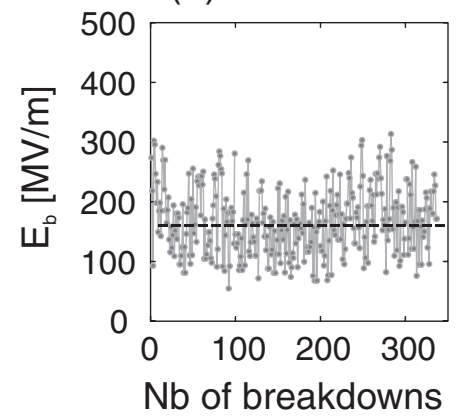

(b) $815^{\circ} \mathrm{C}$

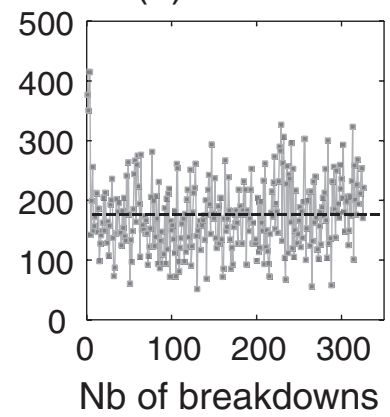

FIG. 7. Effect of $815^{\circ} \mathrm{C}$ heat treatment on $\mathrm{Cu}$.

effect at the emission site on the cathode, driven by field emission, will then dominate.

\section{B. Heat treatment of copper and molybdenum electrodes}

Breakdown tests with heat treated copper electrodes have been carried out. Electrodes are heated ex situ during two hours at $815^{\circ} \mathrm{C}$ in a UHV furnace (pressure below $5 \times$ $10^{-7} \mathrm{mbar}$ ), and then mounted into the spark test chamber. The saturated field is slightly higher, but by less than $10 \%$ as shown in Fig. 7. Larger increases have already been reported, with breakdown fields around $250 \mathrm{MV} / \mathrm{m}$ [24]. It has not been possible to reproduce such improvements in our setup. But in the study cited above, electrodes were specifically prepared by diamond turning to obtain a mirror

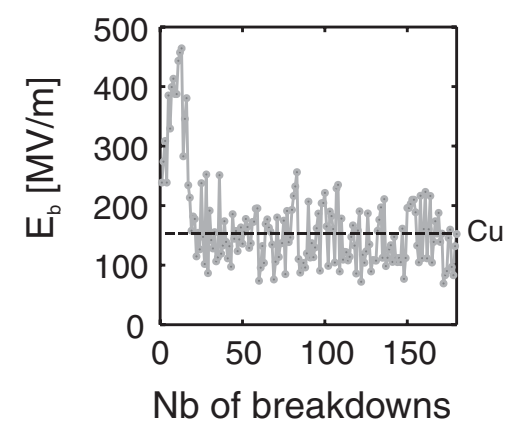

FIG. 8. Conditioning curve of superficially oxidized copper.
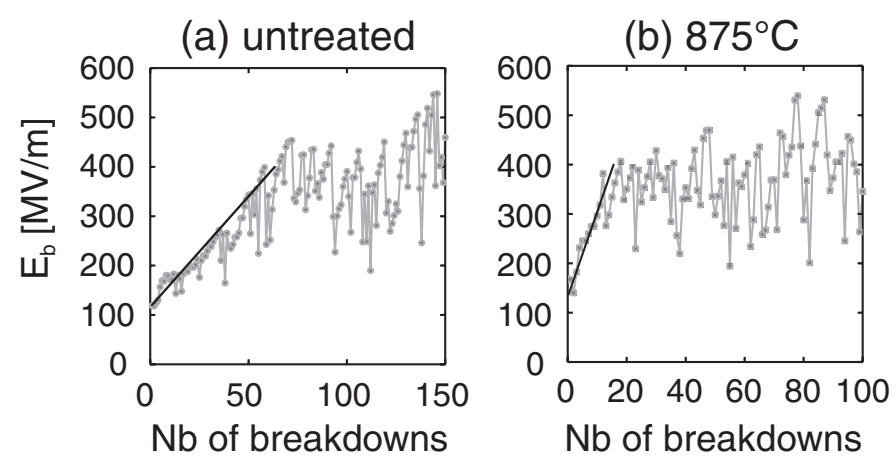

finish, and the geometrical configuration of the setup is also different.

A slight superficial oxidation of copper electrodes surprisingly leads to a significant improvement in the breakdown field (Fig. 8). Tip and sample were simply oxidized by blowing hot ambient air on their surfaces, until their colors changed to brown and then to purple. $\mathrm{A} \mathrm{Cu}_{2} \mathrm{O}$ layer is produced at the surface in this way, and the color of such cuprous oxide thin films is known to be directly related to the thickness of the $\mathrm{Cu}_{2} \mathrm{O}$ layer [25]. The thickness of a purple surface layer is estimated to be around $15 \mathrm{~nm}$. Cuprous oxide $\mathrm{Cu}_{2} \mathrm{O}$ is a $p$-type semiconductor, with a band gap energy of $2.17 \mathrm{eV}$ and with an electron affinity of $3.2 \mathrm{eV}$ [26]. The energy needed to extract an electron from the valence band is thus $5.37 \mathrm{eV}$, which is higher than the work function of pure copper $(4.65 \mathrm{eV})$. This specific property of the copper oxide could be one of the possible causes of the improvement in $E_{b}$, since field emission is lowered for a given field. However, such energy values are measured on perfectly clean surfaces, and we deal here with surfaces which were previously exposed to air. The electric field at the metal surface could also be reduced for a given voltage, due to the presence of the oxide layer on top which acts as a dielectric in a capacitor. As observed in Fig. 8, after 15-20 breakdowns the oxide layer is likely removed and one finds again the standard behavior of copper.

The conditioning speed of Mo can be much improved by a heat treatment. Like copper, the Mo sample is heated ex situ at the nominal temperature in the UHV furnace, and is then exposed to ambient air for about 30 min during transfer and mounting into the spark test chamber. While 60 sparks are necessary to reach a breakdown field of $400 \mathrm{MV} / \mathrm{m}$ with untreated Mo [Fig. 9(a)], only 15 sparks are needed with heated Mo at $875^{\circ} \mathrm{C}$ during 2 hours [Fig. 9(b)]. Treatments at 1000 and $1200^{\circ} \mathrm{C}$ can further reduce the duration of the conditioning phase down to 12 and 10 sparks, respectively [Figs. 9(c) and 9(d)]. The saturated field is not affected by the treatments, remaining around $400 \mathrm{MV} / \mathrm{m}$.

As confirmed by x-ray photoelectron spectroscopy (XPS) measurements [7], the improvement in conditioning

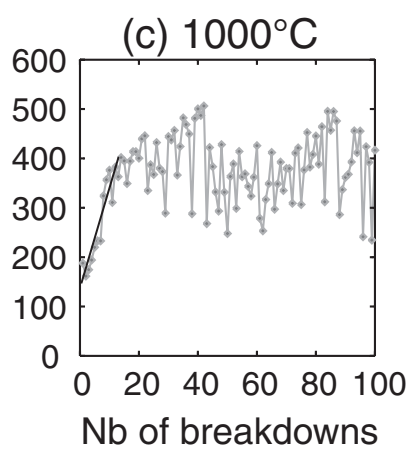

FIG. 9. Improvement in conditioning speed of Mo due to heat treatments. 


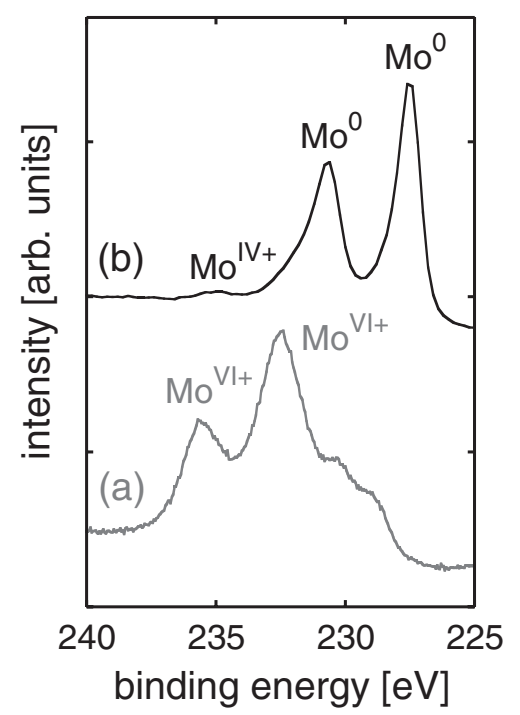

FIG. 10. Mo $3 d$ multiplet photoemission spectra measured on: (a) untreated Mo; (b) Mo heated at $875^{\circ} \mathrm{C}$ in UHV, transferred to the XPS system after a $30 \mathrm{~min}$ air exposure. The spectra are vertically shifted for clarity.

speed can be attributed to the reduction of Mo oxides at the sample surface due to heating (Fig. 10). Whereas a thin film of copper oxides increases the breakdown field of copper, the presence of molybdenum oxides lowers the breakdown field and slows down the conditioning process of Mo. Although the amount of oxide is very low, the conditioning of heated samples is not immediate. A few sparks are still needed to reach the saturated breakdown field, due to the probable presence of field emitters at the surface. Since oxidation of electrodes reduces their conditioning speed, the exposure time to air of the electrodes after the heat treatment has to be minimized. Tests have been done and show that exposures up to eight hours after the treatment do not produce any decrease in the conditioning speed. The energy available in each breakdown $(0.86 \mathrm{~J}$ at $400 \mathrm{MV} / \mathrm{m}$, for example) has probably also an effect on the conditioning process, since a minimum amount of energy is necessary to remove the oxides. Trials with different capacitors to study such an effect are planned.

In order to avoid recrystallization, accelerating structures cannot be heated at too high temperatures and the temperature of the heating has thus to be chosen carefully. As observed with hardness measurements (Vickers test) made on these samples, molybdenum recrystallization occurs beyond $875^{\circ} \mathrm{C}$ for a two hours treatment. Since deformations and embrittlement of structures are more likely if the metal recrystallizes, $875^{\circ} \mathrm{C}$ is a good choice for Mo heat treatment.

\section{Surface treatments of copper electrodes}

The dc conditioning process has been used for many years [27], and is believed to be related to modifications of the electrode surface state due to sparks: melting of fieldemitting protrusions, smoothing, cleaning, removal of surface oxides and adsorbed impurities, for example $[7,28,29]$. As a consequence, the conditioning can be modified depending on the surface preparation.

Since high power rf tests are mainly focused on copper accelerating structures, the effect of different surface treatments on $\mathrm{Cu}$ electrodes has been studied in this dc setup. The standard sample cut from a rolled sheet has been compared to three other samples: a milled sample with low roughness, a chemically polished sample with SUBU (a mixture of sulfamic acid, hydrogen peroxide, $n$-butanol and ammonium citrate [30]), and an electropolished sample [30]. The typical thickness of material removed with the two latter treatments is around $20 \mu \mathrm{m}$.

It is found that the different treatments only affect the first two or three breakdowns. After that, all samples behave in the same way, with an average breakdown field around $170 \mathrm{MV} / \mathrm{m}$ and an average field enhancement factor $\beta$ of around 50. Typically the only differences are seen on the $\beta$ value before the first breakdown, and on the first breakdown field. The results are given in Table I. Values are given with a rather large range, because of the significant spread of the measured data. One sees that the milled and the electropolished samples behave similarly to the standard sample. The results of the chemically polished sample are slightly worse, with a larger $\beta$ and a lower first breakdown field. In any case, the benefit of a careful surface preparation is rapidly erased by a few sparks. This rapid damaging effect is probably less important in an rf accelerating structure, since breakdowns are distributed over a much larger surface. In the dc setup, sparks are repeatedly produced on the same spot, typically $100 \mu \mathrm{m}$ in diameter according to postexperiments scanning electron microscope inspections [6].

Samples of copper and copper alloys machined by electrical discharge machining (EDM) in water have also been tested. They show extremely long conditioning time, typically of several hundreds of breakdowns [31]. EDM, being a thermal machining technique, is known to affect and modify the machined material up to $50 \mu \mathrm{m}$ under the surface [32]. The roughness obtained after machining and the induced surface modifications are the cause of such long conditioning processes. The rf tests conducted with chemically etched Glidcop have also shown that submicron size alumina particles, which are revealed at the surface

TABLE I. Effects of surface treatments on $\mathrm{Cu}$ samples.

\begin{tabular}{lcc}
\hline \hline & $\begin{array}{c}\beta \text { before } \\
\text { 1st spark }\end{array}$ & $\begin{array}{c}\text { 1st breakdown } \\
\text { field }[\mathrm{MV} / \mathrm{m}]\end{array}$ \\
\hline Rolled sheet (reference) & $15-20$ & $200-400$ \\
Milled sample & 20 & $300-500$ \\
Chemically polished sample & $25-30$ & $150-200$ \\
Electropolished sample & $15-20$ & $300-400$ \\
\hline \hline
\end{tabular}


after the etching process, play a significant role in the triggering of breakdowns [33].

\section{Breakdown rate of copper and molybdenum electrodes}

Whereas a high breakdown field is an important requirement for the material of rf accelerating structures, a low breakdown rate (BDR) at a given field is equally important for a practical exploitation of the accelerator. Furthermore, the rf experiments have shown that, for many applications, the information about the breakdown field is only meaningful if the corresponding BDR is also measured. The dc BDR measurements are lengthy (typically seven seconds per attempt) and therefore only possible at high breakdown probability $\left(>10^{-4}\right)$.

In typical dc BDR measurements, attempts giving breakdowns occur stochastically in time during the experimental run, but often come in groups. This feature is also observed in rf experiments, especially at high breakdown rate. Figure 11(a) shows the dc breakdown probability as a function of the applied field for $\mathrm{Cu}$ and Mo electrodes. Both samples were previously conditioned. Results of $30 \mathrm{GHz}$ rf tests [34] are also plotted on the same figure, but against the surface field and not against the accelerating field.

The field at BDR just below 1 is higher than the average saturation field reported in Fig. 5, since the surface is not systematically modified or potentially damaged at each attempt. The BDR measured at the saturated field is around $10^{-2}$. A similar trend is observed both in rf and dc tests: the breakdown probability increases approximately exponentially with the applied field. But taking into account the experimental error bars, data points can be fitted with other types of curves as well. Data are preferentially fitted with power curves here $\left(\mathrm{BDR} \sim E^{k}\right)$ rather than with exponential curves $\left(\mathrm{BDR} \sim e^{k E}\right)$, because of the unphysical $\mathrm{BDR} \neq 0$ at $E=0$ with the latter [35]. The $k$ values of power fits in the dc case range from 10 to 15 for $\mathrm{Cu}$ and from 30 to 35 for Mo, whereas $k$ is around 30 for $\mathrm{Cu}$ and 20 for Mo in the rf case [3,35]. Since breakdowns occur at a much higher field with Mo than with $\mathrm{Cu}$ in dc tests, these slopes are easier to be compared against a normalized field, as shown in Fig. 11(b). For each set of data, the field is normalized with the value at which the breakdown probability is equal to 1 . Although the general trend is the same, it is difficult to compare dc and rf slopes and to have a detailed interpretation of these differences. We are probably at the limit of what can be reasonably compared between these two different kinds of experiments.

\section{CONCLUSION}

The dc breakdown experiments can easily provide measurements of quantities such as breakdown fields and breakdown rates which are also made with rf cavities. Even if these quantities depend on the specific features of the dc setup, these experiments could nevertheless help selecting the best material for the production of rf accelerating structures. For the data set existing so far, the breakdown field ranking of the tested materials corresponds to that found in rf. Obviously, the ranking obtained indicates that a comprehensive model of breakdown must include many physical quantities. Stainless steel shows some interesting properties, with a very high saturated field and a fast conditioning process. A careful surface preparation can also be of importance, as demonstrated with heat treatments of Mo. On the other hand, in our setup the benefits of surface treatments on $\mathrm{Cu}$ electrodes are rapidly destroyed by the damaging effects of a few sparks. More generally, the conditioning speed is clearly related to the initial surface state of the electrodes, but no effect has been observed on the average breakdown field reached after the conditioning phase. Breakdown rate experiments were conducted in dc as well and show a steep dependence on the applied field just as in the rf case. Such dc experiments will continue in parallel to the rf structures testing, and will hopefully add further understanding about the breakdown (a)

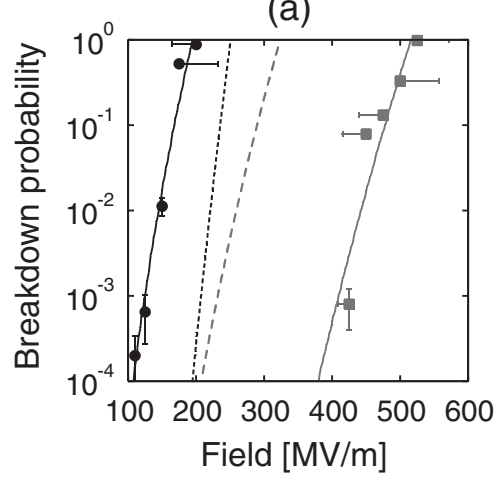

(b)

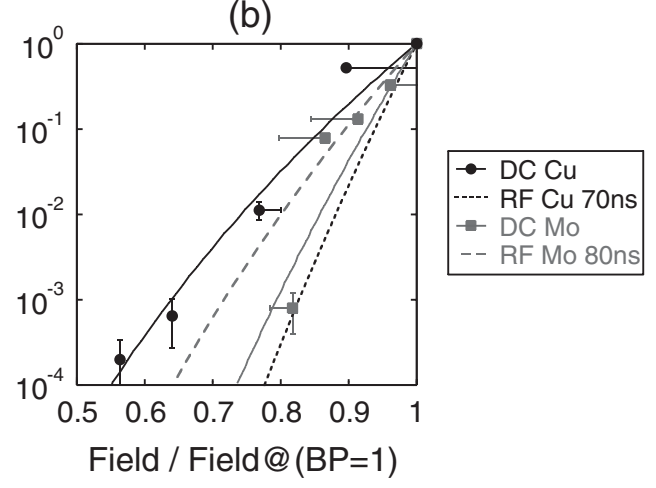

FIG. 11. Breakdown probability as a function of the applied electric field. Comparison of the de results with $\mathrm{rf} 30 \mathrm{GHz}$ results for $\mathrm{Cu}$ and Mo: (a) vs field; (b) vs normalized field at breakdown probability $=1$. 
mechanism.

[1] J.-P. Delahaye, in Proceedings of the 18th Particle Accelerator Conference, New York, 1999 (IEEE, New York, 1999), p. 250.

[2] G. Guignard et al., CERN Report No. CERN-2000-008, 2000.

[3] W. Wuensch, in Proceedings of the 4th Asian Particle Accelerator Conference, Indore, 2007 (RRCAT, Indore, India, 2007), p. 544.

[4] W. Wuensch, in Proceedings of the 11th European Particle Accelerator Conference, Genoa, 2008 (EPS-AG, Genoa, Italy, 2008), p. 2922.

[5] M. Kildemo, Nucl. Instrum. Methods Phys. Res., Sect. A 530, 596 (2004).

[6] M. Kildemo, S. Calatroni, and M. Taborelli, Phys. Rev. ST Accel. Beams 7, 092003 (2004).

[7] T. Ramsvik, S. Calatroni, A. Reginelli, and M. Taborelli, Phys. Rev. ST Accel. Beams 10, 042001 (2007).

[8] M. Taborelli, G. Arnau-Izquierdo, S. Calatroni, S. Heikkinen, T. Ramsvik, S. Sgobba, and W. Wuensch, in Proceedings of the 2007 Particle Accelerator Conference, Albuquerque, New Mexico, 2007 (IEEE, Albuquerque, New Mexico, 2007), p. 2197.

[9] R. L. Boxman, D. M. Sanders, and P. J. Martin, Handbook of Vacuum Arc Science and Technology: Fundamentals and Applications (Noyes Publications, Park Ridge, 1995).

[10] P. Slade, The Vacuum Interrupter: Theory, Design, and Application (CRC Press, Boca Raton, 2008).

[11] F. LePimpec, R. Ganter, and R. Betemps, Nucl. Instrum. Methods Phys. Res., Sect. A 574, 7 (2007).

[12] P. Chatterton, Vacuum Breakdown, in: Electrical Breakdown in Gases, edited by J. Meek and J. Craggs (Wiley, New York, 1978).

[13] W. Boyle, P. Kisliuk, and L. Germer, J. Appl. Phys. 26, 720 (1955).

[14] D. Alpert, D. Lee, E. Lyman, and H. Tomaschke, J. Vac. Sci. Technol. 1, 35 (1964).

[15] F. Charbonnier, C. Bennette, and L. Swanson, J. Appl. Phys. 38, 627 (1967).

[16] D. KennethDavies, J. Vac. Sci. Technol. 10, 115 (1973).

[17] C. Scheuerlein and M. Taborelli, Appl. Surf. Sci. 252, 4279 (2006).
[18] S. Heikkinen, S. Calatroni, H. Neupert, and W. Wuensch, in Proceedings of the 10th European Particle Accelerator Conference, Edinburgh, Scotland, 2006 (EPS-AG, Edinburgh, Scotland, 2006), p. 858.

[19] J. Haimson and B. Mecklenburg, in Proceedings of the 2007 Particle Accelerator Conference, Albuquerque, New Mexico, 2007 (Ref. [8]), p. 2532.

[20] W. Wuensch, A. Grudiev, S. Heikkinen, I. Syratchev, M. Taborelli, I. Wilson, S. Doebert, and C. Adolphsen, in Proceedings of the 22nd International Linear Accelerator Conference, Luebeck, 2004 (DESY-GSI, Luebeck, Germany, 2004), p. 678.

[21] W. Wuensch et al., in Proceedings of the 10th European Particle Accelerator Conference, Edinburgh, Scotland, 2006 (Ref. [18]), p. 801.

[22] W. Wuensch, C. Achard, S. Doebert, H. Braun, I. Syratchev, M. Taborelli, and I. Wilson, in Proceedings of the 20th Particle Accelerator Conference, Portland, OR, 2003 (IEEE, New York, 2003), p. 495.

[23] P. Kranjec and L. Ruby, J. Vac. Sci. Technol. 4, 94 (1967).

[24] S. Kobayashi, Y. Hashimoto, M. Maeyama, Y. Saito, and Y. Nagai, Vacuum 47, 745 (1996).

[25] H. Derin and K. Kantarli, Appl. Phys. A 75, 391 (2002).

[26] D. Zhang, Y. Liu, Y. Liu, and H. Yang, Physica B (Amsterdam) 351, 178 (2004).

[27] R. Millikan and R. Sawyer, Phys. Rev. 12, 167 (1918).

[28] High Voltage Vacuum Insulation, edited by R. V. Latham (Academic Press, New York, 1995).

[29] W. Diamond, J. Vac. Sci. Technol. A 16, 707 (1998).

[30] C. Benvenuti, S. Calatroni, I. E. Campisi, P. Darriulat, M. A. Peck, R. Russo, and A.-M. Valente, Physica C (Amsterdam) 316, 153 (1999).

[31] T. Ramsvik, S. Heikkinen, S. Calatroni, and M. Taborelli, CERN Report No. CERN-TS-Note-2007-007, https:// edms.cern.ch/document/865930/1 (2007).

[32] G. Cusanelli, A. Hessler-Wyser, F. Bobard, R. Demellayer, R. Perez, and R. Fluekiger, J. Mater. Process. Technol. 149, 289 (2004).

[33] L. Laurent, Ph.D. thesis, University of California, Davis, 2002.

[34] J. Rodriguez, in Proceedings of the 2007 Particle Accelerator Conference, Albuquerque, New Mexico, 2007 (Ref. [8]), p. 3818.

[35] A. Grudiev, in CLIC Breakdown Workshop, CERN, 2008, http://indico.cern.ch/conferenceDisplay.py?confId= 33140. 